\title{
Guideline-recommended therapy, including beta- blocker utilization, in patients with chronic heart failure: results from a Canadian community hospital heart function clinic
}

\author{
This article was published in the following Dove Press journal: \\ Research Reports in Clinical Cardiology \\ 10 June 2016 \\ Number of times this article has been viewed
}

Michael Heffernan

Division of Cardiology, Oakville Trafalgar Memorial Hospital, Oakville, ON, Canada

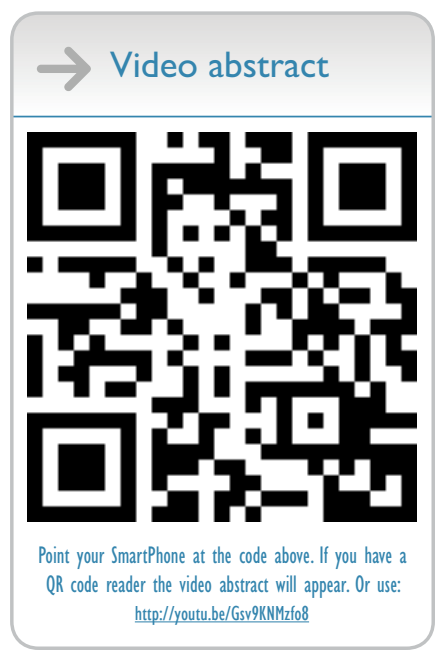

Correspondence: Michael Heffernan Division of Cardiology, Oakville Trafalgar Memorial Hospital, 300I Hospital Gate, Oakville, ON L6M OL8, Canada Email heffernan.cdn@gmail.com

\begin{abstract}
A comprehensive analysis of beta-blocker utilization and other guideline-recommended therapies for the treatment of chronic heart failure in a Canadian community hospital heart function clinic has not been undertaken and was, therefore, the focus of this study. The proportion of patients who would be potential candidates for ivabridine and sacubitril-valsartan therapy as a result of fulfilling the criteria for enrollment in either the Systolic Heart failure treatment with the $I_{\mathrm{f}}$ inhibitor ivabradine Trial (SHIFT) study (left-ventricular ejection fraction [LVEF] $>35 \%$, sinus rhythm, New York Heart Association II-IV) or the Prospective Comparison of angiotensin receptor-neprilysin inhibitor (ARNI) with angiotensin-converting enzyme inhibitor (ACEI) to determine impact on global Mortality and Morbidity in Heart Failure (PARADIGM-HF) study (LVEF $<40 \%$, New York Heart Association II-IV, glomerular filtration rate $>30 \mathrm{~mL} / \mathrm{min}$ ), was also assessed. A retrospective cross-sectional analysis was carried out in all 371 patients treated in this community heart function clinic for at least a 12-month period. The patients were elderly (mean age $74 \pm 13.3$ years) and predominately male (61.5\%) with symptomatic (82.5\%) moderate left-ventricular dysfunction (LVEF $45.4 \% \pm 15.6 \%$ ). A substantial proportion of the patients also had a diagnosis of atrial fibrillation (52.8\%). The total use of beta blockers exceeded $87 \%$, while $100 \%$ of patients without a documented contraindication or intolerance to a beta blocker received therapy. Adherence to other guideline-recommended pharmacotherapies specifically for heart failure with reduced left ventricular ejection was high: $86.1 \%$ of the eligible patients were treated with an ACEI/angiotensin receptor blocker and $61.9 \%$ received a mineralcorticoid receptor antagonist. We determined that $13.7 \%$ of the complement of this heart function clinic could be expected to benefit from ivabridine therapy based on the results of the SHIFT study. In addition, $28.3 \%$ of the clinic patients would be eligible for the initiation of sacubitril-valsartan based on the PARADIGM-HF criteria.
\end{abstract}

Keywords: chronic heart failure, community hospital, heart function clinic, beta blocker, ivabridine, neprilysin inhibitor

\section{Introduction}

Beta blockers have been shown to reduce mortality in patients with congestive heart failure and impaired left-ventricular systolic function. ${ }^{1-4}$ Long-term treatment in patients with impaired left-ventricular (LV) function can reduce the symptoms of heart failure, improve the clinical status of patients, and enhance the quality of life..$^{5-12}$

One advantageous mechanism of beta-blocker therapy in patients with heart failure $(\mathrm{HF})$ is its ability to reduce the resting heart rate. Several studies have established that an elevated resting heart rate is a prognostic factor for cardiovascular events and mortality 
in healthy individuals ${ }^{13,14}$ and in patients with hypertension, ${ }^{15}$ myocardial infarction, ${ }^{16}$ stable coronary artery disease, ${ }^{17,18}$ and HF. ${ }^{19,20}$ Subanalyses of randomized controlled trials in which patients with HF were treated with beta blockers demonstrated a direct relationship between the degree of resting heart rate reduction and a decline in mortality and other major cardiovascular events. ${ }^{20-22}$ In addition, a metaregression analysis of beta-blocker HF trials has shown that the magnitude of survival benefit seen with beta blockers is associated with the level of heart rate reduction and not with the dosage of beta-blocker administered. ${ }^{23}$ Heart rate reduction, therefore, may be an important treatment target for patients with HF.

Ivabridine is an $I_{\mathrm{f}}$ current inhibitor and mediates a reduction in heart rate through its action on this channel in pacemaker cells of the sinus node ${ }^{24}$ and has been shown to benefit patients with HF. Inhibition of the $I_{\mathrm{f}}$ channel results in a reduced sodium and potassium ion flow, which delays the slow diastolic depolarization phase of the sinus node cell action potential, thus lowering the heart rate. The exclusive heart-rate lowering properties of ivabridine were assessed in patients with heart failure with a reduced left-ventricular ejection fraction (HFrEF) in the systolic heart failure treatment with the $I_{\mathrm{f}}$ inhibitor ivabradine trial (SHIFT) study. ${ }^{25}$ The primary composite endpoint of cardiovascular death or hospital admission for worsening HF was significantly reduced in those patients receiving ivabridine. ${ }^{26}$ Ivabridine has now been incorporated into the HF practice guidelines in Europe, ${ }^{27} \mathrm{UK},{ }^{28}$ and more recently, in Canada. ${ }^{29}$

The aim of this study was to perform an extensive review of pharmacologic and device utilization, with a focus on beta-blocker therapy, in a single-center Canadian community hospital heart function clinic since this has not been undertaken previously. In addition, this study sought to determine the proportion of patients in a typical community heart function clinic who would potentially benefit from the addition of relatively new pharmacological therapies available for the treatment of heart failure, namely ivabridine and sacubitril-valsartan.

\section{Methods}

\section{Study population}

In this analysis, we performed a retrospective cross-sectional analysis of all patients followed in the Oakville Trafalgar Memorial Hospital's (OTMH) outpatient heart function clinic in Oakville, ON, Canada, as of April 30, 2014. Only patients treated in the clinic for at least a 12-month period were included in the analysis to allow the titration of pharmacotherapy and implantation of device therapy, if indicated. Patients were assessed with respect to beta-blocker use and dose, demographics, clinical history, comorbidities, and resting heart rate. A subgroup of patients within the clinic who would have met the inclusion criteria for enrollment in the SHIFT study ${ }^{25}$ (left-ventricular ejection fraction [LVEF] $>35 \%$, sinus rhythm, New York Heart Association [NYHA] II-IV) and the Prospective Comparison of angiotensin receptor-neprilysin inhibitor (ARNI) with angiotensin-converting enzyme inhibitor (ACEI) to determine impact on global Mortality and Morbidity in Heart Failure (PARADIGM-HF) study $^{30}$ (LVEF < 40\%, NYHA II-IV, glomerular filtration rate $[\mathrm{GFR}]>30 \mathrm{~mL} / \mathrm{min}$ ) were also analyzed. A review of the adherence to other guideline-recommended pharmacotherapies was also carried out. Patients with implantable cardiodefibrillators and pacemakers were included in the overall analysis. Since this was a review of all patients who were followed in the clinic for at least 1 year and meeting the diagnosis of chronic heart failure (CHF), there were no designated exclusion criteria.

This study conformed to the principles outlined in the 1964 Declaration of Helsinki, and approval from the OTMH Ethics Committee was obtained before initiating this study. A waiver of patient consent was granted from the OTMH Ethics committee for this retrospective analysis.

\section{Data collection}

Data were collected retrospectively from patient charts in the OTMH's heart function clinic. All patients meeting the diagnosis of CHF based on the Canadian Cardiovascular Society guidelines were included. General information, including patient demographics, cardiac history, and previous cardiac investigations, was obtained from the clinic charts. The etiology of CHF was classified as ischemic or nonischemic based on the patient's clinical history and ancillary investigations. Functional assessments were categorized according to the NYHA classification. Prescribed cardiac medications, including beta blockers and their dose, ACEI/angiotensin receptor blocker (ARB), antiplatelet agents, anticoagulants, lipid-lowering therapy, digoxin, diuretics, mineralcorticoid receptor antagonists (MRAs), and antiarrhythmics, were obtained from the patient's clinic chart. Data from patients not treated with a beta blocker due to a contraindication or intolerance were recorded, and the reason for the absence of therapy was documented. Follow-up information on vital status, all-cause admissions and heart failure admissions were obtained from the patient's clinic chart and OTMH's record. Resting heart rates were assessed by the patient's 12-lead electrocardiogram performed during the routine visits to the clinic. LVEFs were determined through the biplane disk 
summation method (Simpson's rule) by two-dimensional echocardiography or by a multigated acquisition scan.

\section{Statistical analysis}

Patient characteristics are shown as the absolute number of patients and percentages, median and quartiles, or mean and standard deviation as appropriate. Patients meeting the SHIFT trial criteria were stratified by heart rate with their most recent heart rates either $\geq 70 \mathrm{bpm}$ or $<70 \mathrm{bpm}$. An additional group was stratified with their most recent heart rates either $\geq 75 \mathrm{bpm}$ or $<75 \mathrm{bpm}$. The groups were compared using the chi-square test or Fisher's exact test and Student's $t$-test or Mann-Whitney $U$-test, as appropriate. The outcomes of death, all-cause admission to hospital, and HF admission to hospital were analyzed for the whole cohort and those meeting the SHIFT criteria. Unadjusted 23-month survival of the entire cohort was estimated by the Kaplan-Meier method. In addition, unadjusted 23-month survival stratified by heart rate and LVEF were estimated by the Kaplan-Meier method.

\section{Results}

In total, 371 patients who were active participants in the OTMH's heart function clinic for 12 months preceding April 30,2014 , were assessed with respect to their treatment dosages of beta blockers, their demographic and clinical profiles, and their resting heart rate. An assessment of other guidelinerecommended pharmacotherapies and device therapy was also carried out.

Table I Baseline demographic and clinical characteristics

\begin{tabular}{ll}
\hline Age (years) & $74 \pm 13.3$ \\
Sex (male) & $228(61.5)$ \\
Body mass index $\left(\mathrm{kg} / \mathrm{m}^{2}\right)$ & $28.8 \pm 6.7$ \\
Ischemic heart disease & $127(34.2)$ \\
Hypertension & $219(59.0)$ \\
Diabetes & $104(28.0)$ \\
Stroke & $35(9.4)$ \\
Atrial fibrillation & $196(52.8)$ \\
Creatinine clearance $(\mathrm{mL} / \mathrm{min})$ & $66.6 \pm 41.0$ \\
Hemodialysis & $13(3.5)$ \\
LVEF (\%) & $45.4 \pm 15.6$ \\
Heart rate (bpm) & $69 \pm 12$ \\
Systolic blood pressure $(\mathrm{mmHg})$ & $126 \pm 19$ \\
Diastolic blood pressure $(\mathrm{mmHg})$ & $71 \pm 10$ \\
NYHA functional class & \\
I & $65(17.5)$ \\
II & $232(62.5)$ \\
III & $69(18.6)$ \\
IV & $5(1.4)$ \\
\hline
\end{tabular}

Note: Data presented as mean standard deviation or number (\%).

Abbreviations: LVEF, left-ventricular ejection fraction; NYHA, New York Heart Association.
Table 2 Baseline pharmacotherapy and device therapy

\begin{tabular}{ll}
\hline & $\begin{array}{l}\text { Overall cohort of HFC } \\
\text { patients (37I) }\end{array}$ \\
Pharmacotherapy & n (\%) \\
Aspirin & $129(34.8)$ \\
Aldosterone receptor antagonists & $147(39.6)$ \\
ACEl & $185(49.9)$ \\
ARB & $117(31.5)$ \\
ACEI/ARB & $298(80.3)$ \\
Beta blocker & $324(87.3)$ \\
Digitalis & $117(29.9)$ \\
Loop diuretics & $285(76.8)$ \\
Antiarrhythmics & $42(11.3)$ \\
Anticoagulants & $189(50.9)$ \\
Device therapy & \\
Pacemaker & $37(10.0)$ \\
Implantable defibrillator & $42(11.3)$ \\
Cardiac resynchronization & $7(1.9)$ \\
\hline Abbrevations ACEl ang
\end{tabular}

Abbreviations: $\mathrm{ACEl}$, angiotensin converting enzyme inhibitor; ARB, angiotensin receptor blocker; HFC, heart function clinic.

Table 1 illustrates the demographic and clinical characteristics of the overall cohort of patients treated in the OTMH's heart function clinic. The optimized pharmacotherapy and device therapy provided to patients in the clinic are outlined in Tables 2 and 3.

A detailed description of beta-blocker use in all patients within the heart function clinic (87.3\%) and those with HFrEF (92.5\%) is displayed in Table 4. Table 4 and Figure 1 also highlight the utilization of the three beta blockers specifically indicated for the treatment of HF among all patients and those with HFrEF. Table 4 and Figure 2 demonstrate the percentage target dose achieved among the beta blockers specifically indicated for the treatment of HF. The mechanism of intolerance for those patients who were not prescribed a beta blocker is also outlined in Table 4.

Of the overall studied population, $51(13.7 \%)$ patients would have fulfilled the criteria for randomization into the SHIFT trial (LVEF $\leq 35 \%$, sinus rhythm, NYHA II-IV). Detailed characteristics of study patients who would have met the inclusion criteria are displayed in Table 5 according to the categories of heart rate. Patients with heart rates $>70$ bpm were younger and exhibited a significantly poorer

Table 3 Guideline-recommended pharmacotherapy in patients with HFrEF

\begin{tabular}{ll}
\hline Pharmacotherapy & Eligible patients* \\
\hline ACEI/ARB & $105 / 122(86.1 \%)$ \\
Beta blockers & $134 / 134(100 \%)$ \\
Mineralcorticoid receptor antagonist & $65 / 105(61.9 \%)$ \\
\hline Note: *Based upon the 20I2 Canadian Heart Failure guidelines and in patients \\
without a contra-indication or intolerance of the medication class. \\
Abbreviations: ACEI, angiotensin converting enzyme inhibitor; ARB, angiotensin \\
receptor blocker; HFrEF, heart failure with reduced left-ventricular ejection.
\end{tabular}

Research Reports in Clinical Cardiology 2016:7 
Table 4 Baseline beta-blocker therapy

\begin{tabular}{|c|c|c|}
\hline & $\begin{array}{l}\text { Overall } \\
\text { clinic cohort }\end{array}$ & $\begin{array}{l}\text { Patients } \\
\text { with HFrEF }\end{array}$ \\
\hline $\begin{array}{l}\text { Patients receiving beta-blocker } \\
\text { therapy }\end{array}$ & $324(87.3)$ & $135(92.5)$ \\
\hline $\begin{array}{l}\text { Patients receiving bisoprolol, } \\
\text { carvedilol, or metoprolol }\end{array}$ & $302(8 I .4)$ & $129(88.3)$ \\
\hline $\begin{array}{l}<50 \% \text { target dose of bisoprolol, } \\
\text { carvedilol, or metoprolol }\end{array}$ & I I $9 / 302(39.4)$ & $65(48.1)$ \\
\hline $\begin{array}{l}\geq 50 \% \text { target dose of bisoprolol, } \\
\text { carvedilol, or metoprolol }\end{array}$ & $126 / 302(4 \mid .7)$ & $70(47.9)$ \\
\hline $\begin{array}{l}100 \% \text { of target dose of bisoprolol, } \\
\text { carvedilol, or metoprolol }\end{array}$ & $57 / 302(18.9)$ & $19(13.0)$ \\
\hline Bisoprolol & $103(27.8)$ & $4 \mathrm{I}(30.4)$ \\
\hline Bisoprolol dose (mg) & $5.6 \pm 3.3$ & $5.2 \pm 2.7$ \\
\hline$\geq 50 \%$ of target dose & $70 / 103(68.0)$ & $26 / 4 \mid(63.4)$ \\
\hline$\geq 100 \%$ of target dose & $26 / 103(25.2)$ & $8 / 4 \mid(19.5)$ \\
\hline Carvedilol & $91(24.5)$ & $52(38.5)$ \\
\hline Carvedilol dose (mg) & $30.8 \pm 18.7$ & $46.0 \pm 33.2$ \\
\hline$\geq 50 \%$ of target dose & $50 / 91(55.0)$ & $27 / 52(51.9)$ \\
\hline$\geq 100 \%$ of target dose & $|4 / 9|(\mid 5.4)$ & $8 / 52(15.4)$ \\
\hline Metoprolol & $108(29.1)$ & $36(26.7)$ \\
\hline Metoprolol dose (mg) & $100.2 \pm 59.7$ & $89.6 \pm 55.2$ \\
\hline$\geq 50 \%$ of target dose & 63/I08 (58.3) & 17/36 (47.2) \\
\hline$\geq 100 \%$ of target dose & $17 / 108(15.7)$ & $3 / 36(8.3)$ \\
\hline Patients intolerant to beta blockers & $47(12.7)$ & II (7.5) \\
\hline \multicolumn{3}{|l|}{ Reasons for intolerance } \\
\hline Hypotension & $\mathrm{I}(0.3)$ & - \\
\hline Fatigue & I $(0.3)$ & - \\
\hline Bradycardia & $5(1.4)$ & $2(1.4)$ \\
\hline COPD & $9(2.4)$ & $5(3.4)$ \\
\hline Asthma & $5(1.4)$ & - \\
\hline Decompensated HF & $12(3.2)$ & $2(1.4)$ \\
\hline Others & $18(4.9)$ & $2(1.4)$ \\
\hline
\end{tabular}

Note: Data presented as mean standard deviation or number (\%). Abbreviation: HFrEF, heart failure with reduced left-ventricular ejection; COPD, chronic obstructive pulmonary disease; $\mathrm{HF}$, heart failure.

NYHA functional class. For those patients who had a heart rate $<70 \mathrm{bpm}$, there was a trend toward a higher rate of betablocker use $(P=0.13)$. When stratifying patients with heart rates $\geq 75 \mathrm{bpm}$ or $<75 \mathrm{bpm}$ (Table 6 ), patients with heart rates $\geq 75 \mathrm{bpm}$ had a significantly poorer NYHA functional class $(P=0.009)$ and a significantly less beta-blocker use $(P=0.028)$.

Tables 7 and 8 highlight the 23-month outcomes of patients who would have fulfilled the SHIFT criteria when stratified with a resting heart rate $<70 \mathrm{bpm}$ or $\geq 70 \mathrm{bpm}$. There was no difference in mortality between the groups. There was a trend for a reduction in HF and all-cause admissions for patients with a heart rate $<70 \mathrm{bpm}$. An additional analysis of outcomes was also carried out when stratifying patients with a resting heart rate $<75 \mathrm{bpm}$ or $\geq 75 \mathrm{bpm}$. There was a significant decrease in the combination of death and HF admission $(P=0.016)$ and death and all-cause admission $(P=0.020)$. In addition, there was a trend toward a decrease in mortality $(P=0.14)$ in patients with a heart rate $<75 \mathrm{bpm}$.

A total of 105 (28.1\%) patients in this community heart function clinic would have met criteria for enrollment in the PARADIGM-HF study (LVEF $<40 \%$, NYHA II-IV, GFR $>30 \mathrm{~mL} / \mathrm{min}$ ) and, therefore, met criteria for the initiation of sacubitril-valsartan.

Admission to OTMH for any cause was assessed from May 1, 2014, to March 20, 2016, and is displayed in Table 9. Patients admitted to hospital for any cause over this time period, were significantly older and had a worse NYHA functional class. Clinic patients admitted with a primary diagnosis of HF over this same time period are highlighted in Table 10. This population had a significantly poorer NYHA functional class $(P=0.0004)$ and were older $(P=0.030)$ than study patients who were not admitted with heart failure.

Mortality was assessed over a 23-month period of all patients within the clinic as of April 30, 2014. The overall mortality rate over this period was $20.2 \%$ (Figure 3 ). Mortality stratified by heart rate $<70 \mathrm{bpm}$ and $\geq 70 \mathrm{bpm}$ and LVEF $<50 \%$ and $\geq 50 \%$ is displayed in Figure 4 .

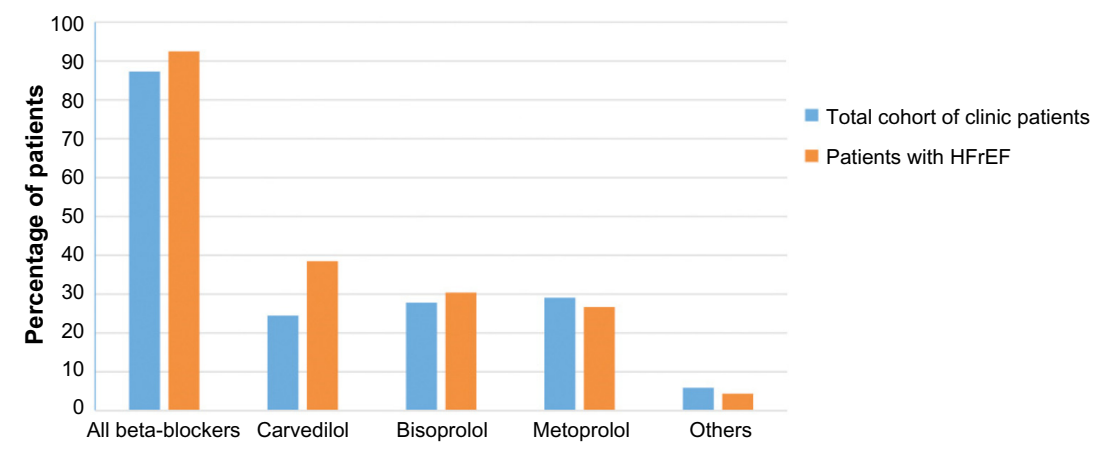

Figure I Beta-blocker use in heart function clinic study patients.

Abbreviation: HFrEF, heart failure with reduced left-ventricular ejection. 


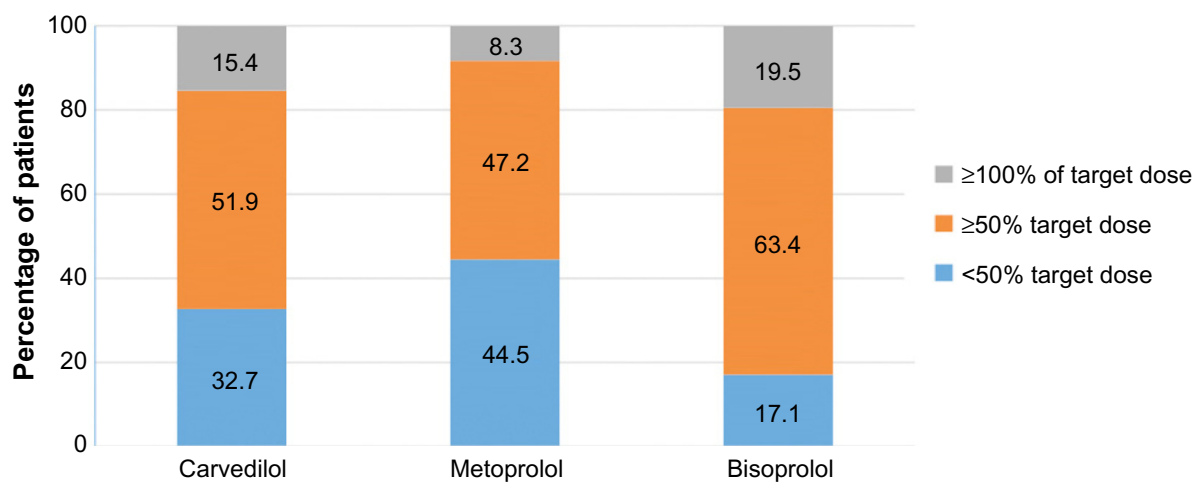

Figure 2 Target dose achieved for beta blockers prescribed to study patients.

\section{Discussion}

This study provides a unique analysis regarding the clinical, demographic, pharmacotherapy and device therapy provided to the full complement of patients in a Canadian community hospital heart function clinic. Overall, the patients in this clinic were elderly (mean age $74 \pm 13.3$ years) and male $(61.5 \%)$ with symptomatic (82.5\%) moderate left-ventricular dysfunction (LVEF 45.4\% $\pm 15.6 \%$ ) (Table 1). A substantial proportion of the patients also had a diagnosis of atrial fibrillation (AF) (52.8\%).

A review of the pharmacotherapy provided to the patients in this clinic demonstrated that physician adherence to treatment guidelines for heart failure was high. Overall, patients receiving ACEI/ARBs, beta blockers, and MRAs represented $80.3 \%, 87.3 \%$, and $39.6 \%$ of the study population, respectively (Tables 2 and 3). These values represent the pharmacotherapy

Table 5 Baseline demographic and clinical characteristics and pharmacotherapy of patients fulfilling SHIFT criteria

\begin{tabular}{|c|c|c|c|c|}
\hline & $\begin{array}{l}\text { Overall cohort } \\
\text { fulfilling select SHIFT } \\
\text { criteria }(n=5 \text { I) }\end{array}$ & $\begin{array}{l}\text { Heart rate } \\
<70 \mathrm{bpm} \\
(\mathrm{n}=32)\end{array}$ & $\begin{array}{l}\text { Heart rate } \\
\geq 70 \mathrm{bpm} \\
(\mathrm{n}=19)\end{array}$ & $P$-value \\
\hline Age (years) & $73.6 \pm 12.9$ & $76.5 \pm 11.1$ & $68.7 \pm 14.4$ & 0.034 \\
\hline Sex (male) & $39(76.5)$ & $24(75.0)$ & $15(79.0)$ & 1.00 \\
\hline Body mass index $\left(\mathrm{kg} / \mathrm{m}^{2}\right)$ & $27.3 \pm 6.1$ & $27.5 \pm 6.3$ & $26.3 \pm 6.2$ & 0.85 \\
\hline Creatinine clearance (mL/min) & $61.3 \pm 44.1$ & $59.8 \pm 46.9$ & $63.9 \pm 39.8$ & 0.76 \\
\hline Heart rate (bpm) & $68 \pm 11$ & $61 \pm 6$ & $79 \pm 10$ & NA \\
\hline Systolic blood pressure $(\mathrm{mmHg})$ & $120 \pm 16$ & $122 \pm 15$ & $116 \pm 18$ & 0.22 \\
\hline Diastolic blood pressure $(\mathrm{mmHg})$ & $68 \pm 9$ & $67 \pm 8$ & $70 \pm 10$ & 0.31 \\
\hline LVEF (\%) & $27.5 \pm 5.4$ & $27.8 \pm 5.1$ & $27.0 \pm 6.0$ & 0.61 \\
\hline \multicolumn{5}{|l|}{ NYHA functional class } \\
\hline II & $38(74.5)$ & $27(84.4)$ & II (57.9) & 0.039 \\
\hline III & II (2I.6) & $5(15.6)$ & $6(31.6)$ & \\
\hline IV & $2(3.9)$ & 0 & $2(10.5)$ & \\
\hline Ischemic etiology of CHF & $29(56.9)$ & $20(62.5)$ & $9(47.4)$ & 0.29 \\
\hline Hypertension & $24(47.1)$ & $13(40.6)$ & II (57.9) & 0.23 \\
\hline Diabetes & $19(37.3)$ & $12(37.5)$ & $7(36.8)$ & 0.96 \\
\hline ACEI/ARB & $42(82.4)$ & $28(87.5)$ & 14 (73.7) & 0.27 \\
\hline Eplerenone & I (2.0) & $I(3.1)$ & 0 & 1.00 \\
\hline Aldactone & $25(49.0)$ & $15(46.9)$ & $10(52.6)$ & 0.69 \\
\hline Digoxin & $10(19.6)$ & $7(21.9)$ & $3(15.8)$ & 0.73 \\
\hline$I C D$ & $16(31.4)$ & $12(37.5)$ & $4(2 I .1)$ & 0.22 \\
\hline PPM & $2(3.9)$ & I (3.I) & I (5.3) & 1.00 \\
\hline Total beta-blocker use & $49(96.1)$ & $32(100.0)$ & $17(89.5)$ & 0.13 \\
\hline Carvedilol/bisoprolol/metoprolol & $48(94.1)$ & $31(96.9)$ & $17(89.5)$ & 0.55 \\
\hline Carvedilol/bisoprolol/metoprolol $\geq 50 \%$ target dose & $24 / 48(50.0)$ & $|7 / 3|(54.8)$ & $7 / 17(4 \mid .2)$ & 0.37 \\
\hline Carvedilol/bisoprolol/metoprolol $100 \%$ target dose & $7 / 48(14.6)$ & $5 / 31(16.1)$ & $2 / 17(11.8)$ & 1.00 \\
\hline
\end{tabular}

Note: Data presented as mean standard deviation or number (\%).

Abbreviations: ACEI, angiotensin converting enzyme inhibitor; ARB, angiotensin receptor blocker; CHF, chronic heart failure; ICD, implantable cardiodefibrillator; LVEF, left-ventricular ejection fraction; NA, not applicable; NYHA, New York Heart Association; PPM, permanent pacemaker; SHIFT, systolic heart failure treatment with the $I_{\mathrm{f}}$ inhibitor ivabradine trial. 
Table 6 Baseline demographic and clinical characteristics and pharmacotherapy of patients fulfilling SHIFT criteria

\begin{tabular}{|c|c|c|c|c|}
\hline & $\begin{array}{l}\text { Overall cohort } \\
\text { fulfilling select SHIFT } \\
\text { criteria }(n=51)\end{array}$ & $\begin{array}{l}\text { Heart rate } \\
<75 \mathrm{bpm} \\
(\mathrm{n}=42)\end{array}$ & $\begin{array}{l}\text { Heart rate } \\
\geq 75 \mathrm{bpm} \\
(\mathrm{n}=9)\end{array}$ & $P$-value \\
\hline Age (years) & $73.6 \pm 12.9$ & $74.9 \pm 12.0$ & $67.6 \pm 15.8$ & 0.12 \\
\hline Sex (male) & $39(76.5)$ & $30(71.4)$ & $9(100.0)$ & 0.067 \\
\hline Body mass index $\left(\mathrm{kg} / \mathrm{m}^{2}\right)$ & $27.3 \pm 6.1$ & $27.4 \pm 5.7$ & $26.9 \pm 8.1$ & 0.83 \\
\hline Creatinine clearance $(\mathrm{mL} / \mathrm{min})$ & $61.3 \pm 44.1$ & $59.2 \pm 44.7$ & $70.7 \pm 42.4$ & 0.48 \\
\hline Heart rate (bpm) & $68 \pm 11$ & $64 \pm 7$ & $86 \pm 9$ & NA \\
\hline Systolic blood pressure $(\mathrm{mmHg})$ & $120 \pm 16$ & $122 \pm 15$ & $112 \pm 20$ & 0.11 \\
\hline Diastolic blood pressure $(\mathrm{mmHg})$ & $68 \pm 9$ & $68 \pm 9$ & $70 \pm 12$ & 0.63 \\
\hline LVEF (\%) & $27.5 \pm 5.4$ & $27.6 \pm 5.0$ & $26.8 \pm 7.3$ & 0.68 \\
\hline \multicolumn{5}{|l|}{ NYHA functional class } \\
\hline II & $38(74.5)$ & $34(81.0)$ & $4(44.4)$ & 0.009 \\
\hline III & II (2I.6) & $8(19.0)$ & $3(33.3)$ & \\
\hline IV & $2(3.9)$ & 0 & $2(22.2)$ & \\
\hline Ischemic etiology of CHF & $29(56.9)$ & $24(57.1)$ & $5(55.6)$ & 1.00 \\
\hline Hypertension & $24(47.1)$ & $19(45.2)$ & $5(55.6)$ & 0.75 \\
\hline Diabetes & $19(37.3)$ & $15(35.7)$ & $4(44.4)$ & 0.71 \\
\hline ACEI/ARB & $42(82.4)$ & $35(83.3)$ & 7 (77.8) & 0.65 \\
\hline Eplerenone & I (2.0) & $\mathrm{I}(2.4 \mathrm{I})$ & 0 & 1.00 \\
\hline Aldactone & $25(49.0)$ & $20(47.6)$ & $5(55.6)$ & 0.73 \\
\hline Digoxin & $10(19.6)$ & $9(21.4)$ & I (II.I) & 0.67 \\
\hline$I C D$ & $16(31.4)$ & $15(35)$. & I (II.I) & 0.24 \\
\hline PPM & $2(3.9)$ & $2(4.8)$ & 0 & 1.00 \\
\hline Total beta-blocker use & $49(96.1)$ & $42(100.0)$ & $7(77.8)$ & 0.028 \\
\hline Carvedilol/bisoprolol/metoprolol & $48(94.1)$ & $4 I(97.6)$ & 7 (77.8) & 0.077 \\
\hline Carvedilol/bisoprolol/metoprolol $\geq 50 \%$ target dose & $24 / 48(50.0)$ & $22 / 4 \mid(53.7)$ & $2 / 7(28.6)$ & 0.42 \\
\hline Carvedilol/bisoprolol/metoprolol 100\% target dose & $7 / 48(14.6)$ & $6 / 41(14.6)$ & I/7 (14.3) & 1.0 \\
\hline
\end{tabular}

Note: Data presented as mean standard deviation or number (\%).

Abbreviations: ACEI, angiotensin converting enzyme inhibitor; ARB, angiotensin receptor blocker; CHF, chronic heart failure; ICD, implantable cardiodefibrillator; LVEF, left-ventricular ejection fraction; NA, not applicable; NYHA, New York Heart Association; PPM, permanent pacemaker; SHIFT, systolic heart failure treatment with the $I_{f}$ inhibitor ivabradine trial.

provided to the entire clinic population, comprised of patients with both preserved and reduced LVEFs. Adherence to guideline-recommended pharmacotherapy specifically for heart failure patients with a reduced left-ventricular ejection was particularly high. In this subgroup of patients, $86.1 \%$ of eligible patients were treated with ACEI/ARBs, $100 \%$ of eligible patients received beta blockers and $61.9 \%$ of eligible patients received MRAs (Table 3).

Our single-center community hospital-based heart function clinic pharmacotherapy performance exceeded the median performance in American and European ambulatory heart failure registries. In the IMPROVE-HF ${ }^{31}$ and PIN$\mathrm{NACLE}^{32}$ studies in which heart failure management was assessed in outpatient American cardiology practices, the rate of ACEI/ARB use was $80 \%$ in IMPROVE-HF and $79 \%$ in PINNACLE. The rate of beta-blocker use was $86 \%$ in IMPROVE-HF and $89 \%$ in PINNACLE. MRA use was not reported in the PINNACLE study; however, in the IMPROVEHF study, MRA use was $34.5 \%$ at baseline and increased to $60.3 \%$ in 24 months following the performance improving

Table 7 Outcome of SHIFT eligible patients stratified by the heart rate of $<70$ bpm versus $\geq 70$ bpm

\begin{tabular}{|c|c|c|c|c|}
\hline Outcomes & $\begin{array}{l}\text { Overall cohort fulfilling } \\
\text { select SHIFT criteria } \\
(n=5 I), n(\%)\end{array}$ & $\begin{array}{l}\text { Heart rate }<70 \text { bpm } \\
(n=32), n(\%)\end{array}$ & $\begin{array}{l}\text { Heart date } \geq 70 \text { bpm } \\
(n=19), n(\%)\end{array}$ & $P$-value \\
\hline Death & $8(15.7)$ & $5(15.6)$ & $3(15.8)$ & I.0 (logrank $P=0.99)$ \\
\hline CHF admission & $7(13.7)$ & $3(9.4)$ & $4(2||)$. & 0.40 \\
\hline All cause admission & $13(25.5)$ & $7(21.9)$ & $6(31.6)$ & 0.51 \\
\hline Death/CHF admission & II (2I.6) & $5(15.6)$ & $6(31.6)$ & 0.29 \\
\hline Death/all cause admission & $16(31.4)$ & $8(25.0)$ & $8(42.1)$ & 0.20 \\
\hline
\end{tabular}

Abbreviations: SHIFT, systolic heart failure treatment with the $I_{\mathrm{f}}$ inhibitor ivabradine trial; $\mathrm{CHF}$, chronic heart failure. 
Table 8 Outcome of SHIFT eligible patients stratified by the heart rate of $<75$ bpm versus $\geq 75$ bpm

\begin{tabular}{|c|c|c|c|c|}
\hline Outcomes & $\begin{array}{l}\text { Overall cohort } \\
\text { fulfilling select } \\
\text { SHIFT criteria } \\
(n=5 \text { I), n (\%) }\end{array}$ & $\begin{array}{l}\text { Heart rate } \\
<75 \text { bpm } \\
(n=42), \\
n(\%)\end{array}$ & $\begin{array}{l}\text { Heart rate } \\
\geq 75 \mathrm{bpm} \\
(\mathrm{n}=9), \\
\mathrm{n}(\%)\end{array}$ & $P$-value \\
\hline Death & $8(15.7)$ & $5(11.9)$ & $3(33.3)$ & $\begin{array}{l}0.14 \\
\text { (logrank } \\
P=0.1 \mathrm{I})\end{array}$ \\
\hline CHF admission & $7(13.7)$ & $4(9.5)$ & $3(33.3)$ & 0.095 \\
\hline $\begin{array}{l}\text { All cause } \\
\text { admission }\end{array}$ & I3 (25.5) & $9(2 \mid .4)$ & $4(44.4)$ & 0.21 \\
\hline $\begin{array}{l}\text { Death/CHF } \\
\text { admission }\end{array}$ & II (2I.6) & $6(14.3)$ & $5(55.6)$ & 0.016 \\
\hline $\begin{array}{l}\text { Death/all cause } \\
\text { admission }\end{array}$ & $16(31.4)$ & $10(23.8)$ & $6(66.7)$ & 0.020 \\
\hline
\end{tabular}

Abbreviations: SHIFT, systolic heart failure treatment with the $I_{f}$ inhibitor ivabradine trial; $\mathrm{CHF}$, chronic heart failure.

interventions from the trial coordinating center. Our adherence to pharmacotherapy also exceeded the performance registries in Europe. In an assessment of CHF management in six European countries, the use of ACEI/ARB, beta blockers, and MRAs were $87 \%, 53 \%$, and $28 \%$, respectively. ${ }^{33} \mathrm{An}$

Table 9 Heart function clinic outpatients all cause hospital admissions (May 2014 to March 2016)

\begin{tabular}{|c|c|c|c|}
\hline Variables & $\begin{array}{l}\text { All cause } \\
\text { admissions } \\
(n=136)\end{array}$ & $\begin{array}{l}\text { No } \\
\text { admissions } \\
(n=235)\end{array}$ & $P$-value \\
\hline Age (years) & $77.5 \pm 10.9$ & $71.2 \pm 14.0$ & $<0.0001$ \\
\hline LVEF (\%) & $47.0 \pm 16.2$ & $44.7 \pm 15.1$ & 0.16 \\
\hline Heart rate (bpm) & $69 \pm 11$ & $68 \pm 12$ & 0.79 \\
\hline \multicolumn{4}{|l|}{ NYHA functional class } \\
\hline I & $8(5.9)$ & $57(24.3)$ & $<0.0001$ \\
\hline ॥ & $88(64.7)$ & $144(6 \mid .3)$ & \\
\hline III & 38 (27.9) & $31(13.2)$ & \\
\hline IV & $2(1.5)$ & $3(1.3)$ & \\
\hline \multicolumn{4}{|l|}{ Beta blocker } \\
\hline Presence & $116(85.3)$ & $208(88.5)$ & 0.37 \\
\hline Absence & $20(14.7)$ & $27(11.5)$ & \\
\hline $\begin{array}{l}\text { Number on beta blockers } \\
\text { that include carvedilol/ } \\
\text { bisoprolol/metoprolol }\end{array}$ & $\mathrm{n}=102$ & $n=200$ & 0.15 \\
\hline$<50 \%$ target dose & $48(47.1)$ & 71 (35.5) & \\
\hline $50 \%-99 \%$ target dose & $37(36.3)$ & $89(44.5)$ & \\
\hline $100 \%$ target dose & $17(16.7)$ & $40(20.0)$ & \\
\hline Meet SHIFT criteria & $13(9.6)$ & $38(16.2)$ & 0.075 \\
\hline \multicolumn{4}{|c|}{ Among those who meet SHIFT criteria } \\
\hline Heart rate $<70 \mathrm{bpm}$ & $7(53.9)$ & $25(65.8)$ & 0.51 \\
\hline Heart rate $\geq 70 \mathrm{bpm}$ & $6(46.1)$ & $13(34.2)$ & \\
\hline \multicolumn{4}{|c|}{ Among those who meet SHIFT criteria } \\
\hline Heart rate $<75$ bpm & $9(69.2)$ & $33(86.8)$ & 0.21 \\
\hline Heart rate $\geq 75$ bpm & $4(30.8)$ & $5(\mid 3.2)$ & \\
\hline
\end{tabular}

Note: Data presented as mean standard deviation or number (\%).

Abbreviations: LVEF, left-ventricular ejection fraction; NYHA, New York Heart Association; SHIFT, systolic heart failure treatment with the $I_{f}$ inhibitor ivabradine trial. assessment of the number and composition of HF clinics in Ontario, Canada, was recently carried out; however, an evaluation of $\mathrm{HF}$ guideline adherence from these centers or another single-center community hospital in Canada has not been reported previously. ${ }^{34}$ Thus far Canadian outpatient HF management data have been limited to abstract publications. ${ }^{35,36}$ This study is the first to reflect the contemporary care in a single nonuniversity-based Canadian outpatient heart function clinic and highlights the excellent adherence to guideline-recommended therapy. The high level of adherence in our clinic is related to the multidisciplinary approach and the incorporation of components that have been shown to be effective in a HF clinic program. This model of care has been shown to prolong survival, reduce hospitalizations, and improve quality of life. ${ }^{37}$ Elements important to patient outcome include a team approach to patient care led by cardiologists with an expertise in heart failure, cardiac nurses, and pharmacists; medication optimization and development of an individualized treatment strategy; patient and family education support; promotion of lifestyle modifications,

Table 10 Heart function clinic outpatients CHF admissions (May 2014 to March 2016)

\begin{tabular}{|c|c|c|c|}
\hline Variables & $\begin{array}{l}\text { CHF } \\
\text { admissions } \\
(n=52)\end{array}$ & $\begin{array}{l}\text { No CHF } \\
\text { admissions } \\
(n=319)\end{array}$ & $P$-value \\
\hline Age (years) & $76.7 \pm 10.7$ & $73.0 \pm 13.6$ & 0.030 \\
\hline LVEF (\%) & $46.7 \pm 16.9$ & $45.3 \pm 15.3$ & 0.54 \\
\hline Heart rate (bpm) & $69.4 \pm 13.4$ & $68.4 \pm 11.5$ & 0.54 \\
\hline \multicolumn{4}{|l|}{ NYHA functional class } \\
\hline I & $2(3.9)$ & $63(19.8)$ & 0.0004 \\
\hline II & $30(57.7)$ & $202(63.3)$ & \\
\hline III & $19(36.5)$ & $50(15.7)$ & \\
\hline IV & I (I.9) & $4(1.2)$ & \\
\hline \multicolumn{4}{|l|}{ Beta blocker } \\
\hline Presence & $47(90.4)$ & $277(86.8)$ & 0.48 \\
\hline Absence & $5(9.6)$ & $42(13.2)$ & \\
\hline $\begin{array}{l}\text { Number on beta blockers } \\
\text { that include carvedilol/ } \\
\text { bisoprolol/metoprolol }\end{array}$ & $\mathrm{n}=42$ & $\mathrm{n}=260$ & \\
\hline$<50 \%$ target dose & $21(50.0)$ & $98(37.7)$ & 0.25 \\
\hline $50 \%-99 \%$ target dose & $13(3 \mid .0)$ & $113(43.5)$ & \\
\hline $100 \%$ target dose & $8(19.0)$ & $49(18.8)$ & \\
\hline Meet SHIFT criteria & $7(13.5)$ & $44(13.8)$ & 0.95 \\
\hline \multicolumn{4}{|c|}{ Among those who meet SHIFT criteria } \\
\hline Heart rate $<70 \mathrm{bpm}$ & $3(42.9)$ & $29(65.9)$ & 0.40 \\
\hline Heart rate $\geq 70 \mathrm{bpm}$ & $4(57.1)$ & $15(34.1)$ & \\
\hline \multicolumn{4}{|c|}{ Among those who meet SHIFT criteria } \\
\hline Heart rate $<75$ bpm & $4(57.1)$ & $38(86.4)$ & 0.095 \\
\hline Heart rate $\geq 75 \mathrm{bpm}$ & $3(42.9)$ & $6(13.6)$ & \\
\hline
\end{tabular}

Note: Data presented as mean standard deviation or number (\%).

Abbreviations: CHF, chronic heart failure; LVEF, left-ventricular ejection fraction; NYHA, New York Heart Association; SHIFT, systolic heart failure treatment with the $I_{f}$ inhibitor ivabradine trial. 


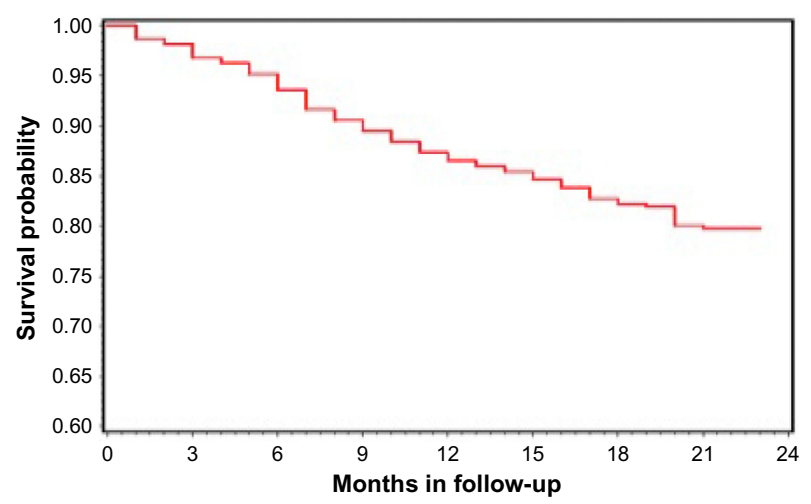

Figure 3 Kaplan-Meier 23-month survival in the OTMH's heart function clinic. Abbreviation: OTMH, Oakville Trafalgar Memorial Hospital.

including the assessment of daily weights, compliance of medication, and avoidance of dietary indiscretion; timely clinic follow-up; and a mechanism to permit patient-initiated access to the clinic for support, if required. ${ }^{37}$

Beta blockers have been shown to reduce cardiovascular morbidity and mortality in patients with congestive heart failure and a reduced LVEF. ${ }^{38-44}$ Several registries and singlecenter studies have assessed the utilization of beta-blocker therapy in outpatient heart function clinics. ${ }^{45-50}$ Despite the effectiveness of beta blockers in the treatment of heart failure, many patients have relative contraindications or experience significant side effects, resulting in a poor compliance or discontinuation. ${ }^{51}$ Even in the landmark clinical trials involving beta-blocker therapy in patients with heart failure, only $80 \%$ of patients were able to achieve the target dose, and a significant proportion of those could not maintain that dose over time..$^{38,39,52,53}$ In our analysis, we were able to show that the overall use of beta blockers exceeded $87 \%$, while $100 \%$ of patients without a documented contraindication or intolerance received therapy (Tables 3 and 4). In comparison to the randomized clinical trials, however, a much smaller proportion of our patient population (18.9\%) were able to tolerate the target doses of the bisoprolol, carvedilol, or metoprolol. Similar shortfalls in achieving guideline-recommended prescription doses have been observed elsewhere outside of the clinical trial setting. ${ }^{45,48,49}$ This clearly demonstrates the clinical need

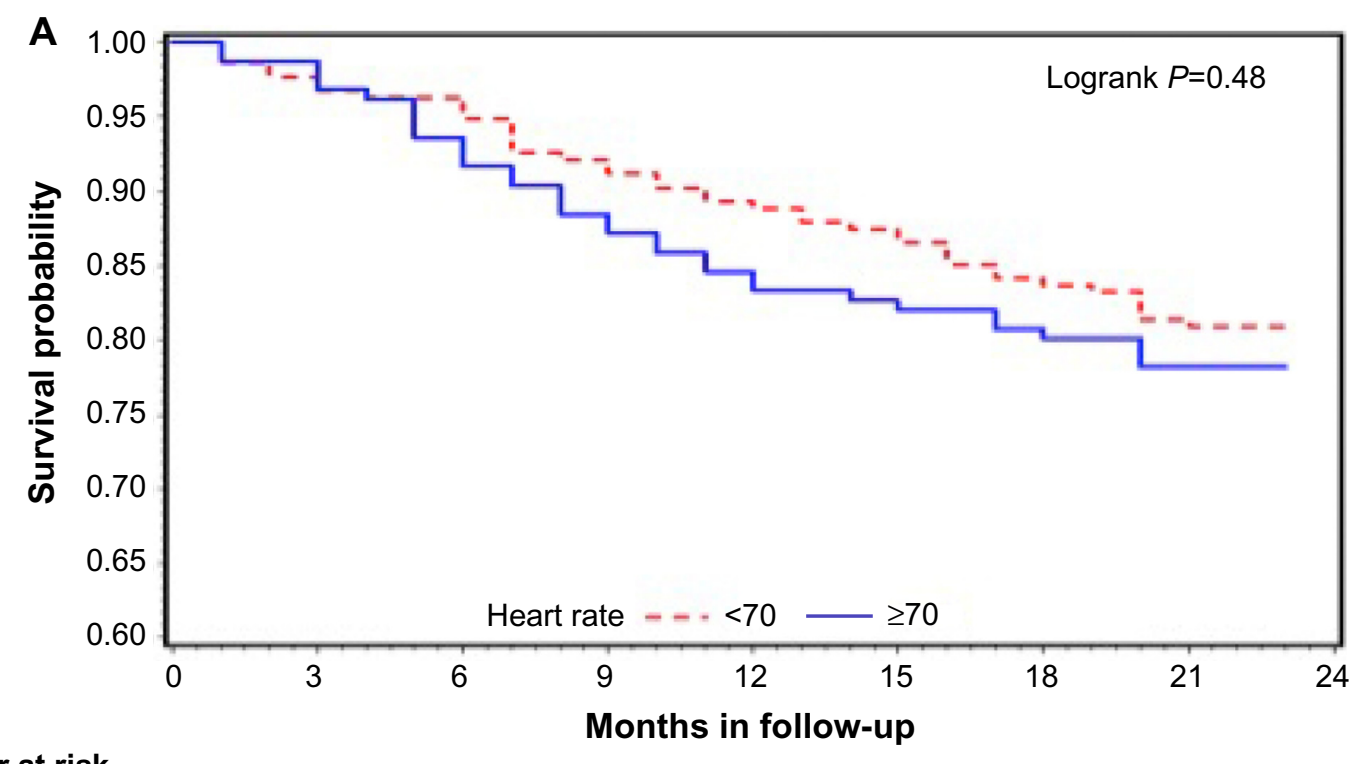

\section{Number at risk}

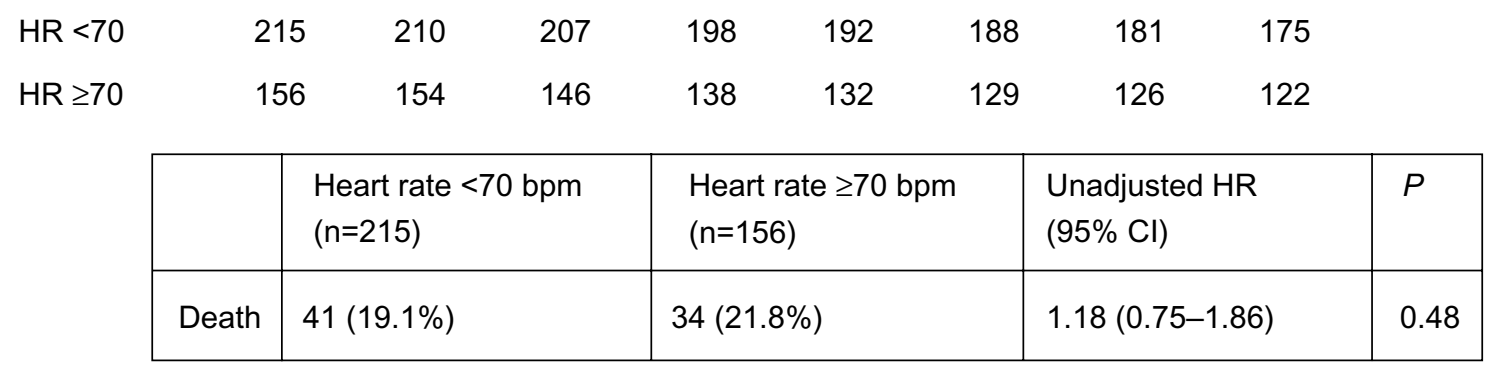

Figure 4 (Continued) 


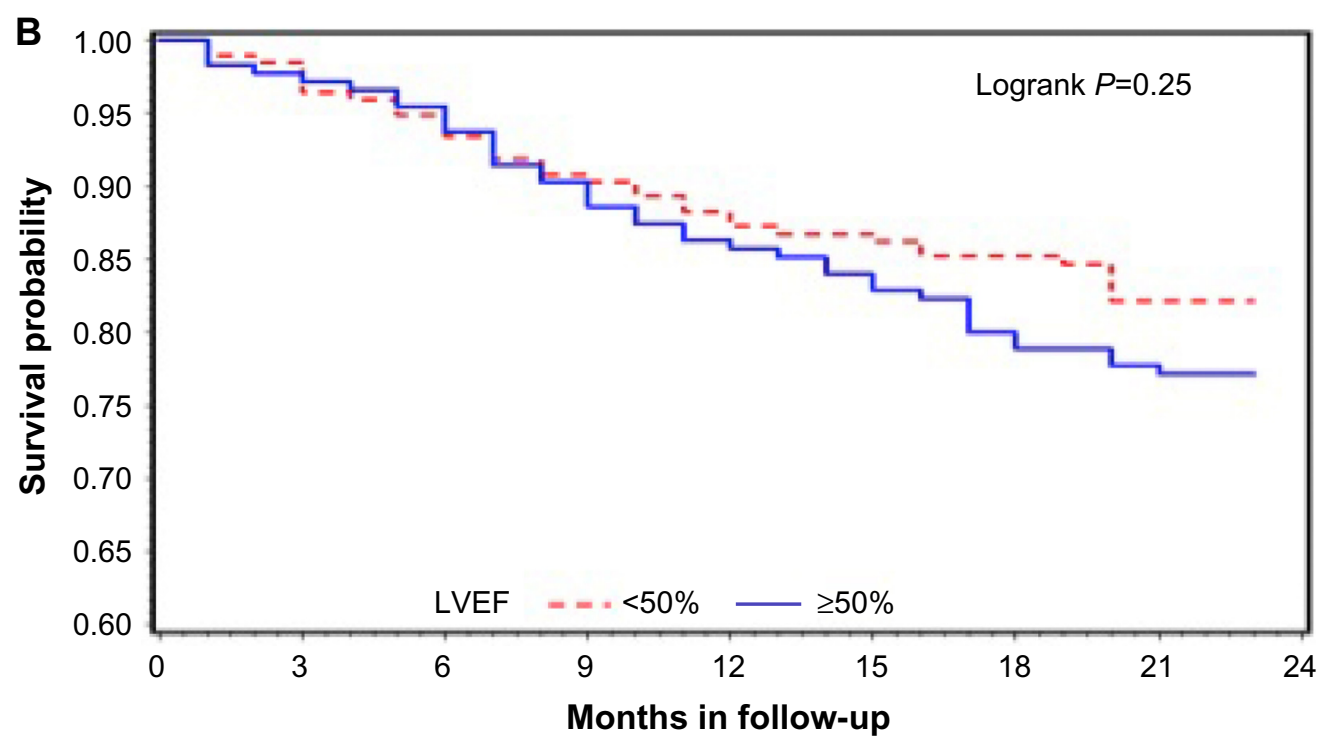

\section{Number at risk}

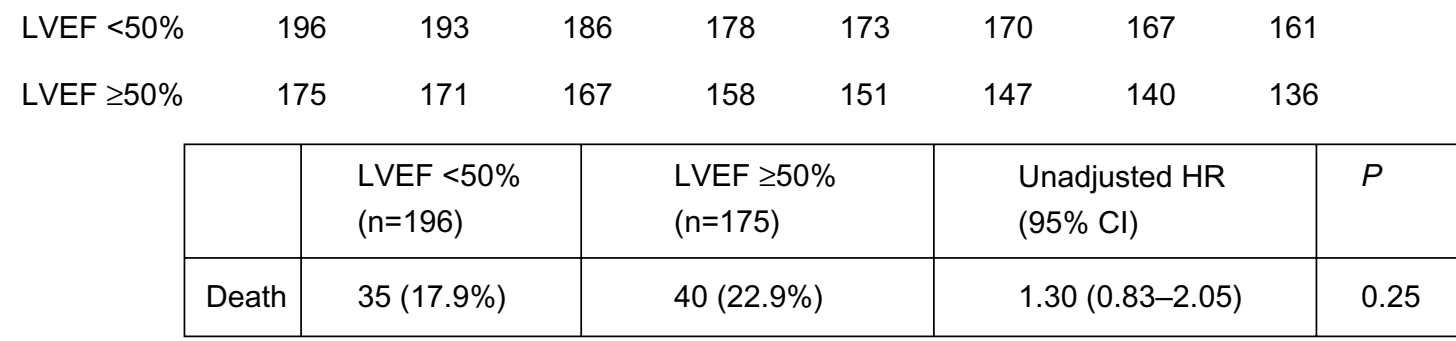

Figure 4 Kaplan-Meier 23-month survival according to the resting heart rate and LVEF.

Notes: (A) Heart rate $<70$ bpm versus $\geq 70$ bpm. (B) LVEF $<50 \%$ versus $\geq 50 \%$. Survival curves not adjusted.

Abbreviation: LVEF, left-ventricular ejection fraction; $\mathrm{Cl}$, confidence interval; $\mathrm{HR}$, heart rate.

for additional heart rate-lowering therapy that is well tolerated and effective in HF.

In the SHIFT study, ivabridine significantly reduced the major cardiovascular outcomes in patients with HF who were in sinus rhythm with a heart rate $>70 \mathrm{bpm} .{ }^{25}$ An analysis of baseline heart rate in SHIFT showed greater benefits, including all-cause mortality, among patients with a baseline heart rate $\geq 75 \mathrm{bpm}$. Based on these results, national and international guidelines from Canada, the UK, and the European Medicines Agency recommend the use of ivabridine for the treatment of patients with HF (NYHA class II-IV) and systolic dysfunction who are in sinus rhythm and receiving guideline-based background therapy, including a beta blocker. ${ }^{27-29}$ There are, however, differences among the three HF guidelines with respect to the clinical phase, background medical therapy, and baseline heart rate that would prompt the initiation of ivabridine. Ivabridine has been approved for the treatment of HF in US, but it has not yet been incorporated into HF practice guidelines. In contrast, the recently updated
Canadian HF guidelines recommend the use of ivabridine for select patients with HF; however, at the moment, it awaits regulatory approval in Canada and has therefore not yet entered into clinical practice.

Based upon the SHIFT criteria it would be expected that a select group of patients in our clinic (13.7\%) would benefit from ivabridine should it be approved for use in Canada. (Tables 5 and 6). In fact, when SHIFT eligible patients were stratified in our cohort with a heart rate of $<75 \mathrm{bpm}$ or $\geq 75 \mathrm{bpm}$, there was a significant reduction in the combination of death/HF admissions and death/all-cause admissions (Tables 7 and 8). The proportion of patients who may be eligible for treatment with ivabridine in this study is similar to the $14 \%$ of patients deemed eligible from a community HF clinic analysis in England. ${ }^{54}$ The number of eligible patients may be even higher as suggested by an analysis from an HF nurse-managed community care initiative in Scotland that demonstrated that $25.4 \%$ of the region's HF patients would meet criteria for ivabridine therapy. ${ }^{55}$ 
Interestingly, a total of $52.8 \%$ of patients in this community hospital-based heart function clinic had a diagnosis of either paroxysmal or permanent AF. The high prevalence of $\mathrm{AF}$ is one major reason why more patients in this study would not meet criteria for the initiation of ivabridine. Since both $\mathrm{AF}$ and $\mathrm{HF}$ occur frequently, it is not surprising that these two conditions coexist among many patients in the clinic. In fact, $\mathrm{HF}$ and $\mathrm{AF}$ share a number of common risk factors, including hypertension, diabetes mellitus, ischemic heart disease, and valvular heart disease ${ }^{56}$ The prevalence of AF in patients with systolic left-ventricular dysfunction and HF has been shown to range from $6 \%$ in patients who are asymptomatic or with minimal symptoms ${ }^{57}$ to between $15 \%$ and $35 \%$ in patients with NYHA class II-IV symptoms. ${ }^{52,58-65}$ In addition, the prevalence of AF has been shown to be greater in HF patients with preserved ejection fractions when compared to those with left-ventricular systolic dysfunction. ${ }^{66,67}$ In our experience, the prevalence of $\mathrm{AF}$ in patients with preserved left-ventricular function ( $\mathrm{EF}>50 \%$ ) was $49.0 \%$ and was, therefore, nearly identical to those with a reduced LVEF. Importantly, adherence to guideline-recommended therapy in our clinic extended also to the treatment of patients with AF. A substantial proportion of eligible patients were prescribed an anticoagulant $(90.8 \%)$ as recommended by the current Canadian Cardiovascular Society guidelines. ${ }^{68}$

An additional pharmacological option for the treatment of heart failure, neprilysin inhibition, was recently approved for use in Canada and has also been incorporated into the updated Canadian Cardiovascular Society HF guidelines. ${ }^{29}$ Inhibition of neprilysin, a neutral endopeptidase, increases the level of vasoactive peptides, including natriuretic peptides, bradykinin, and adrenomedullin. ${ }^{69}$ The products of neprilysin inhibition counter neurohormonal activation that can serve to minimize vasoconstriction, sodium retention, and maladaptive remodeling. When the neprilysin inhibitor sacubitril was combined with the angiotensin receptor antagonist valsartan in the PARADIGM-HF study, there was a significant reduction in the primary endpoint of cardiovascular death or heart failure hospitalization. ${ }^{30}$ In our analysis, $28.3 \%$ of patients within the clinic would have met the criteria for enrollment into the PARADIGM-HF study (LVEF $<40 \%$, NYHA II-IV, GFR $>30 \mathrm{~mL} / \mathrm{min}$ ). This represents a significant proportion of patients who may potentially benefit from sacubitril-valsartan therapy.

Following a diagnosis of HF, mortality estimates are 50\% and $10 \%$ at 5 years and 10 years, respectively. ${ }^{70}$ The mortality of patients treated in a Canadian single-center community HF clinic has not been explored previously. We determined that at our center, 1 -year mortality was $\sim 13 \%$, and the 23 -month mortality was $\sim 20 \%$ (Figure 3 ). Our 1-year value is very similar to the most recently published HF mortality in the province of Ontario, Canada (2007), which estimated an unadjusted 1-year mortality of $15.8 \%{ }^{71}$ among outpatients with HF. We found no statistical difference in mortality when patients were stratified according to their LVEF or resting heart rate (Figure 4); however, this sub-analysis was limited by the small number of patients included in the cohort.

\section{Study limitations}

This cohort study is limited by the characteristic shortcomings of a registry. The value of this registry, however, is the fact that the entire complement of the heart function clinic was included in the analysis. Even patients who would have been typically excluded in a conventional randomized controlled HF study were subjected to study. In addition, we did not exclude patients receiving other chronotropic agents that would be expected to decrease the resting heart rate, such as digitalis and nondihydropyridine calcium channel blockers. Furthermore, unlike a randomized controlled study in which compliance can be assessed, we were unable to ensure the adherence and persistence of pharmacotherapy. Hospitalizations at other institutions apart from the three hospitals that comprise Halton Healthcare Services were not captured, and therefore, the reported event rates may be underestimated. Finally, the heart rate assessments were taken from the 12-lead ECG, as was done in the randomized clinical trials. Ambulatory ECG monitoring may have provided a more comprehensive assessment of heart rate control.

\section{Conclusion}

In this nonrandomized cohort of heart failure patients in a community-based heart function clinic, the adherence to guideline-recommended pharmacotherapy was high. In particular, every patient eligible for beta-blocker therapy received treatment; however, target doses of beta blockers could not be achieved in many patients. A smaller cohort of these patients with a heart rate $\geq 70 \mathrm{bpm}$ and fulfilling the SHIFT trial criteria (LVEF $<35 \%$, sinus rhythm, NYHA II-IV) were identified. Since titration of beta blockers to the doses recommended by landmark clinical trials and national guidelines is often not possible, the addition of a selective heart rate lowering medication, such as ivabridine, may improve the outcomes in a number of patients with HF in this community setting. In addition, this analysis identified a substantial proportion of patients who may also benefit from the initiation of the other relatively new pharmacologic treatment for HF, sacubitril-valsartan. 


\section{Acknowledgments}

The author would like to thank Darlene Lukie and Patricia Franklin for their assistance in data collection for this study. This study was supported by an educational grant from Servier Canada.

\section{Disclosure}

The author served as a speaker and consulting honoraria and/or received research grant support from Amgen, AstraZeneca, Bayer, Boehringer-Ingelheim, Bristol-Myers Squibb, Eisai, Pfizer, Saonfi, and Servier. The author reports no other conflicts of interest in this work.

\section{References}

1. Smith SC Jr, Blair SN, Bonow RO, et al. AHA/ACC scientific statement: AHA/ACC guidelines for preventing heart attack and death in patients with atherosclerotic cardiovascular disease: 2001 update: a statement for healthcare professionals from the American Heart Association and the American College of Cardiology. Circulation. 2001;104(13):1577-1579.

2. Taegtmeyer H, McNulty P, Young ME. Adaptation and maladaptation of the heart in diabetes: part I: general concepts. Circulation. 2002;105(14):1727-1733.

3. Kenchaiah S, Evans JC, Levy D, et al. Obesity and the risk of heart failure. $N$ Engl J Med. 2002;347(5):305-313.

4. He J, Ogden LG, Bazzano LA, Vupputuri S, Loria C, Whelton PK. Risk factors for congestive heart failure in US men and women: NHANES I epidemiologic follow-up study. Arch Intern Med. 2001;161(7):996-1002.

5. Fisher ML, Gottlieb SS, Plotnick GD, et al. Beneficial effects of metoprolol in heart failure associated with coronary artery disease: a randomized trial. J Am Coll Cardiol. 1994;23(4):943-950.

6. Metra M, Nardi M, Giubbini R, Dei Cas L. Effects of short- and longterm carvedilol administration on rest and exercise hemodynamic variables, exercise capacity and clinical conditions in patients with idiopathic dilated cardiomyopathy. J Am Coll Cardiol. 1994;24(7):1678-1687.

7. Olsen SL, Gilbert EM, Renlund DG, Taylor DO, Yanowitz FD, Bristow MR. Carvedilol improves left ventricular function and symptoms in chronic heart failure: a double-blind randomized study. $J \mathrm{Am}$ Coll Cardiol. 1995;25(6):1225-1231.

8. Krum H, Sackner-Bernstein JD, Goldsmith RL, et al. Doubleblind, placebo-controlled study of the long-term efficacy of carvedilol in patients with severe chronic heart failure. Circulation. 1995;92(6):1499-1506.

9. Waagstein F, Bristow MR, Swedberg K, et al; Metoprolol in Dilated Cardiomyopathy (MDC) Trial Study Group. Beneficial effects of metoprolol in idiopathic dilated cardiomyopathy. Lancet. 1993;342(8885):1441-1446.

10. CIBIS Investigators and Committees. A randomized trial of beta blockade in heart failure: the Cardiac Insufficiency Bisoprolol Study (CIBIS). Circulation. 1994;90(4):1765-1773.

11. Packer M, Colucci WS, Sackner-Bernstein JD, et al. Double blind, placebo-controlled study of the effects of carvedilol in patients with moderate to severe heart failure: the PRECISE trial: prospective randomized evaluation of carvedilol on symptoms and exercise. Circulation. 1996;94(11):2793-2799.

12. Colucci WS, Packer M, Bristow MR, et al; US Carvedilol Heart Failure Study Group. Carvedilol inhibits clinical progression in patients with mild symptoms of heart failure. Circulation. 1996;94(11):2800-2806.

13. Jouven X, Empana JP, Buyck JF, et al. Resting heart rate and its changes over years as a risk factor for mortality in the general population: the Paris prospective study I. Eur Heart J. 2007;27:333.
14. Mensink GB, Hoffmeister $H$. The relationship between resting heart rate and all-cause, cardiovascular and cancer mortality. Eur Heart J. 1997;18(9):1404-1410.

15. Gillman MW, Kannel WB, Belanger A, D’Agostino RB. Influence of heart rate on mortality among persons with hypertension: the Framingham study. Am Heart J. 1993;125(4):1148-1154.

16. Hjalmarson A, Gilpin EA, Kjekshus J, et al. Influence of heart rate on mortality after acute myocardial infarction. Am J Cardiol. 1990;65(9):547-553.

17. Fox K, Ford I, Steg PG, et al; BEAUTIFUL investigators. Heart rate as a prognostic risk factor in patients with coronary artery disease and leftventricular systolic dysfunction (BEAUTIFUL): a subgroup analysis of a randomised controlled trial. Lancet. 2008;372(9641):817-821.

18. Kolloch R, Legler UF, Champion A, et al. Impact of resting heart rate on outcomes in hypertensive patients with coronary artery disease: findings from the International Verapamil-SR/Trandolapril Study (INVEST). Eur Heart J. 2008;29(10):1327-1334.

19. Böhm M, Swedberg K, Komajda M, et al; SHIFT Investigators. Heart rate as a risk factor in chronic heart failure (SHIFT): the association between heart rate and outcomes in a randomised placebo-controlled trial. Lancet. 2010;376(9744):886-894.

20. Lechat P, Hulot JS, Escolano S, et al. Heart rate and cardiac rhythm relationships with bisoprolol benefit in chronic heart failure in CIBIS II trial. Circulation. 2001;103(10):1428-1433.

21. Lechat P, Escolano S, Golmard JL, et al. Prognostic value of bisoprolol-induced hemodynamic effects in heart failure during the Cardiac Insufficiency BIsoprolol Study (CIBIS). Circulation. 1997;96(7):2197-2205.

22. Metra M, Torp-Pedersen C, Swedberg K, et al. Influence of heart rate, blood pressure, and beta-blocker dose on outcome and the differences in outcome between carvedilol and metoprolol tartrate in patients with chronic heart failure: results from the COMET trial. Eur Heart J. 2005;26(21):2259-2268.

23. McAlister FA, Wiebe N, Ezekowitz JA, Leung AA, Armstrong PW. Meta-analysis: beta-blocker dose, heart rate reduction, and death in patients with heart failure. Ann Intern Med. 2009;150(11):784-794.

24. Ferrari R, Ceconi C. Selective and specific $I_{\mathrm{f}}$ inhibition with ivabradine: new perspectives for the treatment of cardiovascular disease. Expert Rev Cardiovasc Ther. 2011;9(8):959-973.

25. Swedberg K, Komajda M, Bohm M, et al; SHIFT Investigators. Ivabradine and outcomes in chronic heart failure (SHIFT): a randomised placebo-controlled trial. Lancet. 2010;376(9744):875-885.

26. Fox K, Ford I, Steg PG, et al; BEAUTIFUL Investigators. Relationship between ivabradine treatment and cardiovascular outcomes in patients with stable coronary artery disease and left ventricular systolic dysfunction with limiting angina: a subgroup analysis of the randomized, controlled BEAUTIFUL trial. Eur Heart J. 2009;30(19):2337-2345.

27. McMurray JJ, Adamopoulos S, Anker SD, et al; ESC Committee for Practice Guidelines. ESC guidelines for the diagnosis and treatment of acute and chronic heart failure 2012: the task force for the diagnosis and treatment of acute and chronic heart failure 2012 of the European society of cardiology. developed in collaboration with the heart failure association (HFA) of the ESC. Eur Heart $J$. 2012;33(14):1787-1847.

28. NICE Pathways [homepage on the internet]. London. Available from: http:// pathways.nice.org.uk/pathways/chronic-heart-failure\#path=view\%3A/ pathways/chronic-heart-failure/treatment-for-heart-failure-due-to-leftventricular-systolic-dysfunction.xml\&content=view-index. Accessed April 8, 2016.

29. Howlett JG, Chan M, Ezekowitz JA, et al; Canadian Cardiovascular Society Heart Failure Guidelines Panels. The Canadian cardiovascular society heart failure companion: bridging guidelines to your practice. Can J Cardiol. 2016;32(3):296-310.

30. McMurray J, Packer M, Desai AS, et al; PARADIGM-HF Investigators and Committees. Angiotensin-neprilysin inhibition versus enalapril in heart failure. N Engl J Med. 2014;371(11):993-1004. 
31. Fonarow GC, Albert NM, Curtis AB, et al. Improving evidence-based care for heart failure in outpatient cardiology practices primary results of the registry to improve the use of evidence-based heart failure therapies in the outpatient setting (IMPROVE HF). Circulation. 2010;122(6):585-596.

32. Peterson PN, Chan PS, Spertus JA, et al. Practice-level variation in use of recommended medications among outpatients with heart failure: insights from the NCDR PINNACLE program. Circ Heart Fail. 2013;6(6):1132-1138.

33. Komajda M, Lapuerta P, Hermans N, et al. Adherence to guidelines is a predictor of outcome in chronic heart failure: the MAHLER survey. Eur Heart J. 2005;26(16):1653-1659.

34. Wijeysundera HC, Trubiani G, Abrahamyan L, et al. Specialized multidisciplinary heart failure clinics in Ontario, Canada: an environmental scan. BMC Health Serv Res. 2012;12:236.

35. Arnold JMO, Ignazweski A, Howlett J, et al; Canadian Heart Failure Network Inc. Changes in drug utilization in patients referred to outpatient heart failure clinics in Canada between 1999 and 2007. J Am Coll Cardiol. 2009;53:A166.

36. Howlett JG, Johnstone DE, Sketris I, et al; Improving Cardiovascular Outcomes in Nova Scotia investigators. Identifying opportunities to address the congestive heart failure burden: the Improving Cardiovascular Outcomes in Nova Scotia (ICONS) study. Can J Cardiol. 2003;19(4):439-444.

37. Cooper LB, Hernandez AF. Assessing the quality and comparative effectiveness of team-based care for heart failure who, what, where, when, and how. Heart Fail Clin. 2015;11(3):499-506.

38. The Cardiac Insufficiency Bisoprolol Study II (CIBIS-II): a randomized trial. Lancet. 1999;353(9146):9-13.

39. Effect of metoprolol $\mathrm{CR} / \mathrm{XL}$ in chronic heart failure: metoprolol $\mathrm{CR} / \mathrm{XL}$ randomized intervention trial in congestive heart failure (MERIT-HF). Lancet. 1999;353(9169):2001-2007.

40. Lechat P, Packer M, Chalon S, Cucherat M, Arab T, Boissel JP. Clinical effects of beta-adrenergic blockade in chronic heart failure: a metaanalysis of double-blind, placebo-controlled, randomized trials. Circulation. 1998;98(12):1184-1191.

41. Packer M, Bristow MR, Cohn JN, et al; U.S. Carvedilol Heart Failure Study Group. The effect of carvedilol on morbidity and mortality in patients with chronic heart failure. $N$ Engl $J$ Med. 1996;334(21):1349-1355.

42. Packer M, Coats AJ, Fowler MB, et al; Carvedilol Prospective Randomized Cumulative Survival Study Group. Effect of carvedilol on survival in severe chronic heart failure. $N$ Engl J Med. 2001;344(22):1651-1658.

43. Dargie HJ. Effect of carvedilol on outcome after myocardial infarction in patients with left-ventricular dysfunction: the CAPRICORN randomised trial. Lancet. 2001;357(9266):1385-1390.

44. Cleland JG, Pennell DJ, Ray SG, et al; Carvedilol hibernating reversible ischaemia trial: marker of success investigators. Myocardial viability as a determinant of the ejection fraction response to carvedilol in patients with heart failure (CHRISTMAS trial): randomised controlled trial. Lancet. 2003;362(9377):14-21.

45. Maggioni AP, Dahlström U, Filippatos G, et al; Heart Failure Association of ESC (HFA). EURObservational research programme: the heart failure pilot survey (ESC-HF Pilot). Eur J Heart Fail. 2010;12(10):1076-1084.

46. de Groote P, Isnard R, Assyag P, et al. Is the gap between guidelines and clinical practice in heart failure treatment being filled? Insights from the IMPACT RECO survey. Eur J Heart Fail. 2007;9(12):1205-1211.

47. Fonarow GC, Albert NM, Curtis AB, et al. Incremental reduction in risk of death associated with use of guideline-recommended therapies in patients with heart failure: a nested case-control analysis of IMPROVE HF. J Am Heart Assoc. 2012;1(1):16-26.

48. Komajda M, Follath F, Swedberg K, et al; Study Group on Diagnosis of the Working Group on Heart Failure of the European Society of Cardiology. The EuroHeart failure survey programme - a survey on the quality of care among patients with heart failure in Europe. Part 2: treatment. Eur Heart J. 2003;24(5):464-474.
49. Franke J, Wolter JS, Meme L, et al. Optimization of pharmacotherapy in chronic heart failure: is heart rate adequately addressed? Clin Res Cardiol. 2013;102(1):23-31.

50. Gupta R, Tang WH, Young JB. Patterns of beta-blocker utilization in patients with chronic heart failure: experience from a specialized outpatient heart failure clinic. Am Heart J. 2004;147(1):79-83.

51. Butler J, Khadim G, Belue R, et al. Tolerability to beta-blocker therapy among heart failure patients in clinical practice. $J$ Card Fail. 2003;9(3):203-209.

52. Flather MD, Shibata MC, Coats AJ, et al; SENIORS Investigators. Randomized trial to determine the effect of nebivolol on mortality and cardiovascular hospital admission in elderly patients with heart failure (SENIORS). Eur Heart J. 2005;26(3):215-225.

53. Packer M, Fowler MB, Roecker EB, et al; Carvedilol Prospective Randomized Cumulative Survival (COPERNICUS) Study Group. Effect of carvedilol on the morbidity of patients with severe chronic heart failure: results of the carvedilol prospective randomized cumulative survival (COPERNICUS) study. Circulation. 2002;106(17):2194-2199.

54. Cullington D, Goode KM, Cleland JG, Clark AL. Limited role for ivabridine in the treatment of chronic heart failure. Heart. 2011;97(23):1961-1966.

55. Elder DH, Mohan M, Cochrane L, Charles H, Lang CC. Characterizing patients with chronic heart failure in community care after hospitalization: a potential role for ivabridine. Cardiovasc Ther. 2015;33(3):104-108.

56. Ho KK, Pinsky JL, Kannel WB, Levy D. The epidemiology of heart failure: the Framingham study. J Am Coll Cardiol. 1993;22(4 Suppl A): $6 \mathrm{~A}-13 \mathrm{~A}$.

57. Dries DL, Exner DV, Gersh BJ, Domanski MJ, Waclawiw MA, Stevenson LW. Atrial fibrillation is associated with an increased risk for mortality and heart failure progression in patients with asymptomatic and symptomatic left ventricular systolic dysfunction: a retrospective analysis of the SOLVD trials. Studies of left ventricular dysfunction. J Am Coll Cardiol. 1998;32(3):695-703.

58. van Veldhuisen DJ, Aass H, El Allaf D, et al; MERIT-HF Study Group. Presence and development of atrial fibrillation in chronic heart failure. Experiences from the MERIT-HF study. Eur J Heart Fail. 2006;8(5):539-546.

59. De Ferrari GM, Klersy C, Ferrero P, et al; ALPHA Study Group. Atrial fibrillation in heart failure patients: prevalence in daily practice and effect on the severity of symptoms. Data from the ALPHA study registry. Eur J Heart Fail. 2007;9(5):502-509.

60. Poole-Wilson PA, Swedberg K, Cleland JG, et al; Carvedilol Or Metoprolol European Trial Investigators. Comparison of carvedilol and metoprolol on clinical outcomes in patients with chronic heart failure in the carvedilol or metoprolol European trial (COMET): randomised controlled trial. Lancet. 2003;362(9377):7-13.

61. Corell P, Gustafsson F, Schou M, Markenvard J, Nielson T, Hildebrandt P. Prevalence and prognostic significance of atrial fibrillation in outpatients with heart failure due to left ventricular systolic dysfunction. Eur $J$ Heart Fail. 2007;9(3):258-265.

62. Olsson LG, Swedberg K, Ducharme A, et al; CHARM Investigators. Atrial fibrillation and risk of clinical events in chronic heart failure with and without left ventricular systolic dysfunction: results from the candesartan in heart failure-assessment of reduction in mortality and morbidity (CHARM) program. J Am Coll Cardiol. 2006;47(10):1997-2004.

63. Deedwania PC, Singh BN, Ellenbogen K, Fisher S, Fletcher R, Singh SN. Spontaneous conversion and maintenance of sinus rhythm by amiodarone in patients with heart failure and atrial fibrillation: observations from the veterans affairs congestive heart failure survival trial of antiarrhythmic therapy (CHF-STAT). The Department of Veterans Affairs CHF-STAT Investigators. Circulation. 1998;98(23):2574-2579.

64. Maggioni AP, Latini R, Carson PE, et al; Val-HeFT Investigators. Valsartan reduces the incidence of atrial fibrillation in patients with heart failure: results from the valsartan heart failure trial (Val-HeFT). Am Heart J. 2005;149(3):548-557. 
65. Swedberg K, Olsson LG, Charlesworth A, et al. Prognostic relevance of atrial fibrillation in patients with chronic heart failure on long-term treatment with beta-blockers: results from COMET. Eur Heart J. 2005;26(13):1303-1308.

66. Bhatia RS, Tu JV, Lee DS, et al. Outcome of heart failure with preserved ejection fraction in a population-based study. $N$ Engl J Med. 2006;355(3):260-269.

67. Owan TE, Hodge DO, Herges RM, Jacobsen SJ, Roger VL, Redfield MM. Trends in prevalence and outcome of heart failure with preserved ejection fraction. $N$ Engl J Med. 2006;355(3):251-259.

68. Verma A, Cairns JA, Mitchell LB, et al; CCS Atrial Fibrillation Guidelines Committee. Focused update of the Canadian cardiovascular society guidelines for the management of atrial fibrillation. Can J Cardiol. 2014;30(10):1114-1130.
69. Gori M, Senni M. Sacubitril/valsartan (LCZ696) for the treatment of heart failure. Expert Rev Cardiovasc Ther. 2016;14(2):145-153.

70. Roger VL. Epidemiology of heart failure. Circ Res. 2013;113(6): 646-659.

71. Yeung DF, Boom NK, Guo H, Lee DS, Schultz SE, Tu JV. Trends in the incidence and outcomes of heart failure in Ontario, Canada: 1997 to 2007. CMAJ. 2012;184(14):E765-E773.

\section{Publish your work in this journal}

Research Reports in Clinical Cardiology is an international, peerreviewed, open access journal publishing original research, reports, editorials, reviews and commentaries on all areas of cardiology in the clinic and laboratory. The manuscript management system is completely online and includes a very quick and fair peer-review system.
Visit http://www.dovepress.com/testimonials.php to read real quotes from published authors. 